\title{
miR-1301-3p promotes the proliferation and migration of lung cancer cells via direct repression of polymerase I and transcript release factor
}

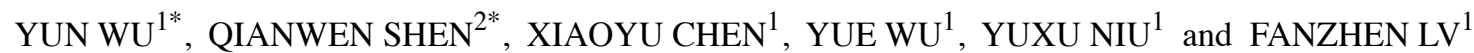 \\ Departments of ${ }^{1}$ Thoracic Surgery and ${ }^{2}$ Radiation Oncology, Shanghai Key Laboratory of Clinical Geriatric Medicine, \\ Huadong Hospital Affiliated with Fudan University, Shanghai 200040, P.R. China
}

Received February 26, 2020; Accepted June 19, 2020

DOI: 10.3892/ol.2020.12149

\begin{abstract}
Aberrant expression of microRNAs (miRNAs or miRs) is associated with a number of human diseases, including lung cancer. Although numerous differentially expressed miRNAs have been identified in lung cancer via microarray and sequencing methods, to the best of our knowledge, only a small portion of these miRNAs have been experimentally verified. In the present study, miR-1301-3p expression levels in lung tumor tissues and lung cancer cells were measured by reverse transcription-quantitative PCR (RT-qPCR) and by analyzing previously published data. Cell Counting Kit- 8 and Transwell assays were used to analyze the function of miR-1301-3p in lung cancer tissues and cells. Bioinformatics analysis, RT-qPCR, western blotting and a dual-luciferase reporter assay were performed to investigate the mechanism of miR-1301-3p in lung cancer cells. It was identified that miR-1301-3p is an upregulated miRNA in lung cancer via analyzing previously published microarray and The Cancer Genome Atlas-lung squamous cell carcinoma project data, and the upregulation of miR-1301-3p was confirmed in collected clinical samples and cells. Inhibition of miR-1301-3p suppressed lung cancer cell proliferation and migration. In addition, miR-1301-3p inhibition upregulated E-cadherin, an epithelial cell maker, and downregulated vimentin, a mesenchymal cell marker. Using bioinformatics analysis, it was revealed that polymerase I and transcript release factor (PTRF) is a target of miR-1301-3p. RT-qPCR, western blotting and dual-luciferase reporter assays
\end{abstract}

Correspondence to: Dr Yuxu Niu or Dr Fanzhen Lv, Department of Thoracic Surgery, Shanghai Key Laboratory of Clinical Geriatric Medicine, Huadong Hospital Affiliated with Fudan University, 221 West Yan'an Road, Shanghai 200040, P.R. China

E-mail: niuyuxu@gmail.com

E-mail: lvfanzhendoc@163.com

*Contributed equally

Key words: microRNA-1301-3p, polymerase I and transcript release factor, migration, lung cancer demonstrated that PTRF is targeted by miR-1301-3p in lung cancer cells. The rescue experiments indicated that silencing PTRF could attenuate the inhibition of cell proliferation and migration induced by miR-1301-3p inhibitor in lung cancer cells. Furthermore, a strong negative correlation between miR-1301-3p and PTRF mRNA was identified in clinical samples. In summary, the present data highlight the involvement of miR-1301-3p in the proliferation and migration of lung cancer cells, indicating that miR-1301-3p may be a promising biomarker for lung cancer.

\section{Introduction}

Globally, lung cancer is the most prevalent cancer type in both males and females, and there were $\sim 1,760,000$ lung cancer-associated mortalities reported in 2018 (1). The majority of diagnosed lung cancer cases are histologically classified as non-small cell lung cancer (NSCLC) (2). NSCLC can be further divided into several subtypes, of which lung adenocarcinoma (LUAD) and lung squamous cell carcinoma (LUSC) are the most common types (3). Although the development of targeted therapy and cancer screening methods have improved the prognosis of patients with lung cancer, lung cancer remains a lethal cancer type for humans, with a 5-year overall survival rate of $<20 \%$ (4). The high proliferative ability and aggressive nature of lung cancer cells is responsible for the poor prognosis of patients (5).

microRNAs (miRNAs or miRs) are short, non-coding molecules expressed in mammal cells (6). miRNAs can interact with the 3'-untranslated region (3'UTR) of target mRNAs, causing mRNA to degrade or for the translation process to stop (7). It is understood that miRNAs control the expression of key genes in multiple signaling pathways to promote normal physiological processes, such as cell differentiation and cell death (8). Dysregulation of miRNAs disrupts signaling networks and contributes to the progression of human diseases, including lung cancer $(9,10)$. Numerous differentially expressed miRNAs have been identified in lung cancer according to several previous studies via microarray methods (11-13). Several miRNAs have been confirmed as key cancer-associated miRNAs in lung cancer via targeting oncogenes and tumor suppressors (14-16). For example, miR-19b 
directly represses protein phosphatase 2 and BIM expression, activates epidermal growth factor receptor signaling, and promotes cell proliferation and apoptosis resistance in lung cancer cells (17). A previous study screened out numerous differentially expressed miRNAs in lung cancer with brain metastasis (13). However, the biological roles and expression patterns of the majority of these miRNAs have not yet been investigated.

The present study analyzed previously published microarray data (13) and identified miR-1301-3p as an upregulated miRNA in lung cancer with brain metastasis. Therefore, the aim was to investigate the expression, biological function and molecular mechanisms of miR-1301-3p in lung cancer cells.

\section{Materials and methods}

Patient samples. A total of 40 lung tumor tissue and matched normal tissue $(>3 \mathrm{~cm}$ away from cancer) samples were obtained from patients (29 male and 11 female, age range 35-80 years; median 55 years) with lung cancer at Huadong Hospital Affiliated with Fudan University (Shanghai, China) between June 2014 and July 2017. All participants provided written informed consent. Lobectomy was performed to resect tumors and matched normal tissues from patients. None of the patients received chemotherapy or radiotherapy before the surgery and they did not have other malignancies. The protocol of the experiments was approved by the Ethical Committee of Huadong Hospital Affiliated with Fudan University (approval no. FDU201406-2). Specimens were snap-frozen in liquid nitrogen and stored in a $-80^{\circ} \mathrm{C}$ refrigerator.

Cell culture and cell transfection. The immortalized lung epithelial cell line BEAS-2B and lung cancer cell lines A549 and H1299 were purchased from the American Type Culture Collection. Cells were maintained in DMEM (Gibco; Thermo Fisher Scientific, Inc.) supplemented with 10\% FBS (Gibco; Thermo Fisher Scientific, Inc.) and $1 \%$ penicillin-streptomycin solution (HyClone; Cytiva) in an incubator at $37^{\circ} \mathrm{C}$ with 5\% $\mathrm{CO}_{2}$. miR-1301-3p inhibitor (5'-GAAGUCACUCCC AGGCAGCUGCAA-3'), miR-1301-3p mimic (5'-UUGCAG CUGCCUGGGAGUGACUUC-3'), miR-negative control (NC) mimic (5'-UUCUCCGAACGUGUCACGUTT-3') and miR-NC inhibitor (5'-CAGUACUUUUGUGUAGUA CAA-3') were obtained from Guangzhou RiboBio Co., Ltd. A549 and H1299 cells were transfected with $50 \mathrm{nM}$ miRNA mimic, inhibitor, miR-NC mimic or miR-NC inhibitor using Lipofectamine 3000 reagent (Invitrogen; Thermo Fisher Scientific, Inc.) according to the manufacturer's protocol. Polymerase I and transcript release factor (PTRF) small interfering RNA (siRNA) (5'-GAGAAGCGCAUGAACAAG CUGTT-3') and control siRNA (5'-UUCUCCGAACGUGUC ACGUTT-3') were obtained from Shanghai GenePharma Co., Ltd. Lipofectamine 3000 was used to transfect $100 \mathrm{nM}$ siRNA into A549 and H1299 cells. After 48 h, the transfection efficiency was determined by western blotting and reverse transcription-quantitative PCR (RT-qPCR).

Cell proliferation and migration assays. The cell proliferation assay was performed with Cell Counting Kit-8 (CCK-8; Dojindo Molecular Technologies, Inc.). At 0, 24, 48 and $72 \mathrm{~h}$ post-transfection, $\mathrm{CCK}-8$ was added to the culture medium and maintained for $2 \mathrm{~h}$. Subsequently, the absorbance at $450 \mathrm{nM}$ was detected by a i-Mark Microplate Reader (Bio-Rad Laboratories, Inc.) to measure the cell number.

The migration capacity of cancer cells was detected with an $8-\mu \mathrm{m}$ pore size Boyden chamber (EMD Millipore). Cells were suspended in DMEM containing 0.5\% FBS (Gibco; Thermo Fisher Scientific, Inc.) and seeded in the upper chamber, and DMEM containing $10 \%$ FBS was added to the lower chamber as the chemoattractant. Following $48 \mathrm{~h}$, cells remaining in the upper side of the chamber were removed, and cells on the other side of the chamber were treated with $4 \%$ formaldehyde followed by crystal violet staining at room temperature for $15 \mathrm{~min}$. Three random fields of $\mathrm{miR}-\mathrm{NC}$ inhibitor + control siRNA, miR-1301-3p inhibitor + control siRNA and miR-1301-3p inhibitor + PTRF siRNA groups were examined with an inverted light microscope (magnification, $\mathrm{x} 40$; Nikon Corporation) and the number of migrated cells was analyzed.

$R T$ - $q P C R$. RNA was collected from cells and tissues using TRIzol (Invitrogen; Thermo Fisher Scientific, Inc.) according to the manufacturer's protocol. First-stranded cDNA was synthesized using M-MLV reverse transcriptase (Invitrogen; Thermo Fisher Scientific, Inc.) with dNTP set (Invitrogen; Thermo Fisher Scientific, Inc.) and Random Hexamers (Invitrogen; Thermo Fisher Scientific, Inc.) according to the manufacturer's protocol. qPCR was performed with a TB Green Premix Ex Taq $^{\text {TM }}$ II kit (Takara Bio, Inc.) on the CFX96 system (Bio-Rad Laboratories, Inc.). The thermocycling conditions were as follows: $95^{\circ} \mathrm{C}$ for $10 \mathrm{sec}$, followed by 40 cycles of $95^{\circ} \mathrm{C}$ for $5 \mathrm{sec}$ and $60^{\circ} \mathrm{C}$ for $30 \mathrm{sec}$. mRNA and miRNA expression were normalized to GAPDH and U6. The $2^{-\Delta \Delta \mathrm{Cq}}$ method (18) was applied to analyze the relative expression of genes. The primer sequences were as follows: E-cadherin forward, 5'-AAAGGC CCATTTCCTAAAAACCT-3' and reverse, 5'-TGCGTTCTC TATCCAGAGGCT-3'; Vimentin forward, 5'-TGCCGTTGA AGCTGCTAACTA-3' and reverse, 5'-CCAGAGGGAGTG AATCCAGATTA-3'; PTRF forward, 5'-AGGTCAGCGTCA ACGTGAAG-3' and reverse, 5'-CCGACTCTTTCAGCGATT TGC-3'; GAPDH forward, 5'-CTGGGCTACACTGAGCAC C-3' and reverse 5'-AAGTGGTCGTTGAGGGCAATG-3'; Stem-loop, 5'-CTCAACTGGTGTCGTGGAGTCGGCAAT TCAGTTGAGGAAGTC-3'; miR-1301-3p forward, 5'-GCC GAGTTGCAGCTGCCTGGGA-3' and reverse, 5'-CTCAAC TGGTGTCGTGGA-3'; and U6 forward, 5'-GCTTCGAGG CAGGTTACATG-3' and reverse, 5'-GCAACACACAACATC TCCCA-3'.

Western blot analysis. Lysates were obtained from cells using RIPA buffer (Thermo Fisher Scientific, Inc.) according to the manufacturer's protocol. The concentration of protein lysates was determined by a BCA protein assay kit (Thermo Fisher Scientific). PTRF (cat. no. 69036; 1:1,000), E-cadherin (cat. no. 14472; 1:1,000) and vimentin (cat. no. 5741; 1:1,000) antibodies were purchased from Cell Signaling Technology, Inc. GAPDH (cat. no. ab8245; 1:5,000) was obtained from Abcam. Horseradish peroxidase (HRP)-conjugated goat anti-mouse (cat. no. ab97040; 1:10,000) and HRP-conjugated goat anti-rabbit (cat. no. ab7090; 1:10,000) were also purchased 

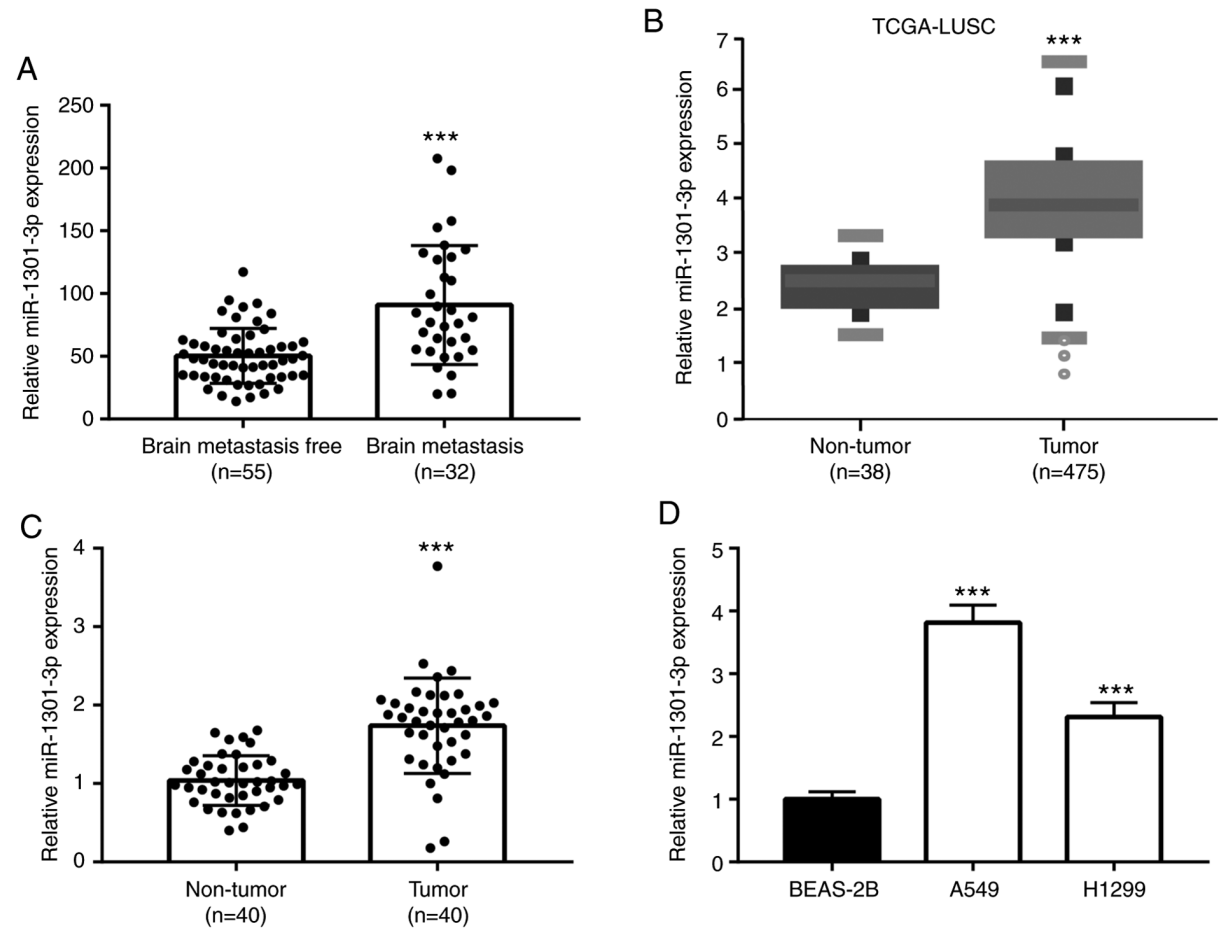

Figure 1. miR-1301-3p is increased in lung cancer. (A) Analysis of miR-1301-3p level in 32 lung tumor samples with brain metastasis and 55 lung tumor samples with non-brain metastasis from previously published microarray data. ${ }^{* * *} \mathrm{P}<0.001$ vs. non-brain metastasis. (B) The expression of miR-1301-3p in LUSC ( $n=475)$ and normal lung tissues $(n=38)$ was analyzed in TCGA-LUSC dataset. ${ }^{* * *} \mathrm{P}<0.001$ vs. non-tumor. (C) The level of miR-1301-3p in 40 pairs of lung tumors and matched normal tissues was measured in the collected samples via RT-qPCR. ${ }^{* * *} \mathrm{P}<0.001$ vs. non-tumor. (D) The level of miR-1301-3p in BEAS-2B, A549 and H1299 cells was measured by RT-qPCR in this study. ${ }^{* * *} \mathrm{P}<0.001$ vs. BEAS-2B cells. RT-qPCR, reverse transcription-quantitative PCR; miR-1301-3p, microRNA-1301-3p; TCGA, The Cancer Genome Atlas; LUSC, lung squamous cell carcinoma.

from Abcam. Proteins $(20 \mu \mathrm{g})$ were separated by $8 \%$ SDS-PAGE, and then transferred to a PVDF membrane. The PVDF membrane was blocked with $5 \%$ non-fat milk for $1 \mathrm{~h}$ at room temperature $\left(25^{\circ} \mathrm{C}\right)$, followed by incubation with the primary antibodies for $1 \mathrm{~h}$ at room temperature and secondary antibodies for $1 \mathrm{~h}$ at room temperature. The membrane was treated with the ECL Western Blotting Substrate (Pierce; Thermo Fisher Scientific, Inc.) to detect blot signals. The bands were quantified using ImageJ v1.52 software (National Institutes of Health).

Bioinformatics analysis. The expression data of miRNAs in lung cancer with brain metastasis $(n=32)$ and those with non-brain metastasis $(\mathrm{n}=55)$ were obtained from previously published data (13). The association between the expression of miR-1301-3p and PTRF in LUAD tissues, LUSC tissues and normal lung tissues was analyzed using the Encyclopedia of RNA Interactomes (ENCORI) software (http://starbase.sysu. edu.cn/) based on The Cancer Genome Atlas (TCGA)-LUAD and TCGA-LUSC datasets (http://starbase.sysu.edu. cn/panMirDiffExp.php) (19). The ENCORI software was also used to evaluate the expression of miR-1301-3p in TCGA-LUSC dataset. The prediction of targets of miR-1301-3p and putative binding sites was performed using TargetScan version 7.2 (http://www.targetscan.org/vert_72/).

Dual-luciferase reporter assay. PTRF 3'UTR was inserted into the pmirGLO vector (Promega Corporation). pmirGLO-PTRF-Mut (mutant) was established via introducing two-point mutations into pmirGLO-PTRF-WT (wild-type). pmirGLO-PTRF-WT or pmirGLO-PTRF-WT were co-transfected with miR-1301-3p mimic or miR-NC mimic using Lipofectamine 3000 (Thermo Fisher Scientific, Inc.) into A549 cells. After $48 \mathrm{~h}$, the luciferase activity was detected with a Dual-Luciferase ${ }^{\circledR}$ Reporter Assay System kit (Promega Corporation). Firefly luciferase activity was normalized to that of Renilla luciferase.

Statistical analysis. Data were analyzed using GraphPad Prism 5.0 (GraphPad Software, Inc.) and presented as mean \pm SD, and $\mathrm{P}<0.05$ was considered to indicate a statistically significant difference. The experiments were repeated in triplicate. Two groups were analyzed by unpaired Student's t-test. Multiple groups were analyzed by one-way analysis of variance with Tukey's post hoc test. The correlation between miR-1301-3p and PTRF expression levels was examined with Pearson's correlation test.

\section{Results}

miR-1301-3p is increased in lung cancer. To investigate the miRNAs involved in metastasis of lung cancer, previously published microarray data (13) were analyzed. It was identified that miR-1301-3p was one of the miRNAs expressed at a significantly higher level in lung tumors with brain metastasis $(n=32)$ compared with those with non-brain metastasis $(n=55)$ (Fig. 1A). Furthermore, the expression of miR-1301-3p was evaluated in TCGA-LUSC dataset, which included expression profile data of 38 normal lung tissues and 475 NSCLC tissues. The results demonstrated that miR-1301-3p was significantly 

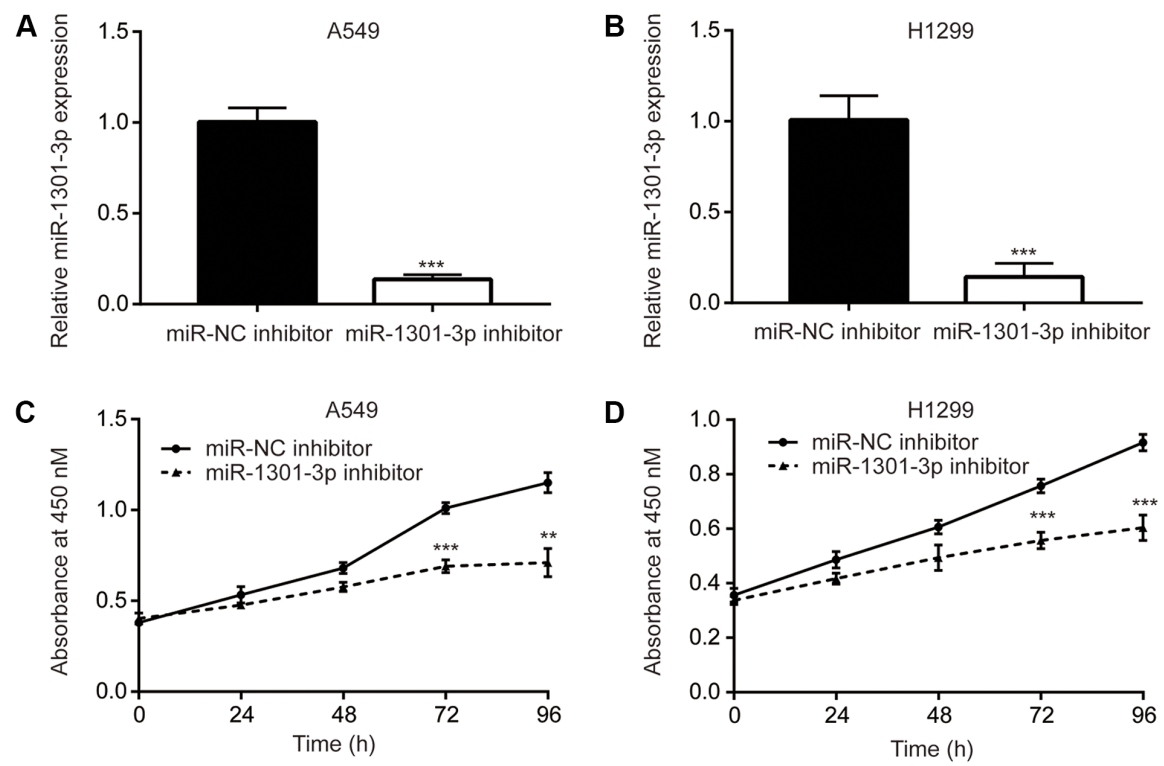

E
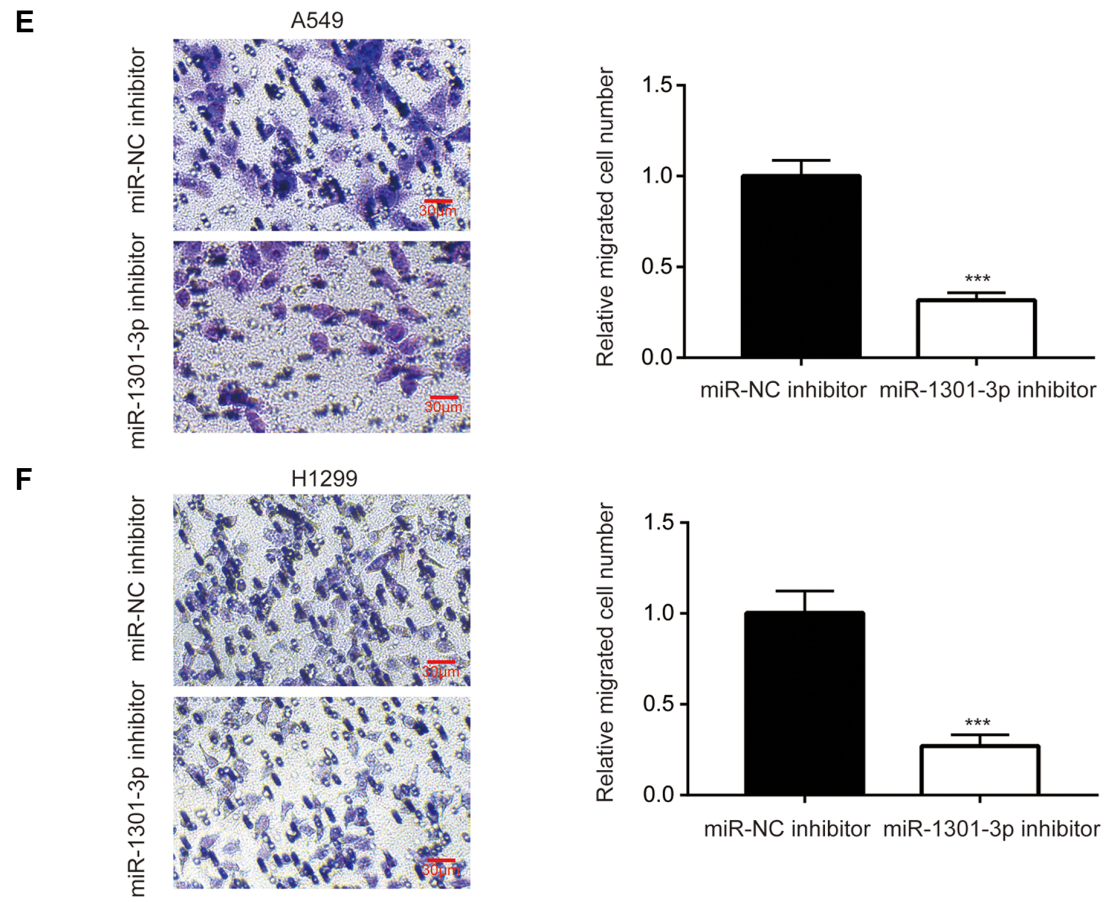

Figure 2. Downregulation of miR-1301-3p negatively affects lung cancer cell proliferation and migration. Reverse transcription-quantitative PCR was performed to measure miR-1301-3p level in (A) A549 and (B) H1299 cells after transfection with miR-1301-3p inhibitor or NC. Via Cell Counting Kit-8 assay, cell proliferation of (C) A549 and (D) H1299 cells following transfection with miR-1301-3p inhibitor or NC was detected. Cell migration of (E) A549 and (F) H1299 cells after transfection of miR-1301-3p inhibitor or NC was measured. ${ }^{* *} \mathrm{P}<0.01,{ }^{* * *} \mathrm{P}<0.001$ vs. miR-NC inhibitor. miR, microRNA; NC, negative control.

upregulated in lung cancer tissues compared with normal lung tissues (Fig. 1B). For validation, 40 pairs of tumors and matched normal samples were collected from patients with lung cancer. RT-qPCR revealed that miR-1301-3p was significantly higher in lung cancer samples compared with matched normal samples (Fig. 1C). Furthermore, it was observed that miR-1301-3p expression was significantly higher in A549 and H1299 cells compared with the immortalized lung epithelial cell line BEAS-2B (Fig. 1D).

Downregulation of miR-1301-3p suppresses lung cancer cell proliferation and migration. miR-1301-3p inhibitor was transfected into lung cancer cell lines A549 and H1299. RT-qPCR was then performed to confirm that miR-1301-3p inhibitor significantly reduced miR-1301-3p expression in A549 and H1299 cells (Fig. 2A and B). miR-1301-3p inhibitor significantly suppressed the proliferation of A549 and H1299 cells at 72 and 96 h (Fig. 2C and D). In addition, Transwell assay demonstrated that miR-1301-3p inhibitor significantly decreased the number of A549 and H1299 cells that migrated through the membrane (Fig. 2E and F). These data demonstrated that miR-1301-3p is associated with lung cancer cell proliferation and migration.

PTRF is suppressed by miR-1301-3p. Using TargetScan, several genes were predicted as targets of miR-1301-3p. It was identified that there was a complementary site for miR-1301-3p on the 3'UTR of PTRF (Fig. 3A). Using ENCORI software, 
A

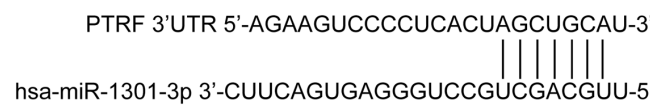

C

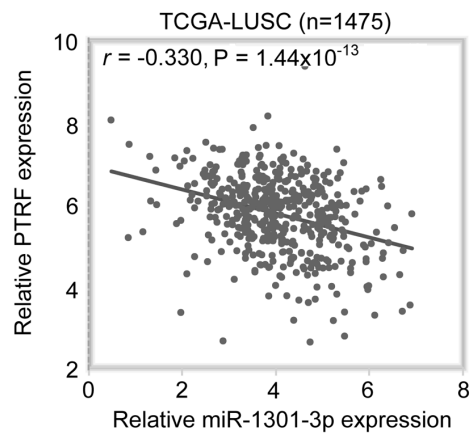

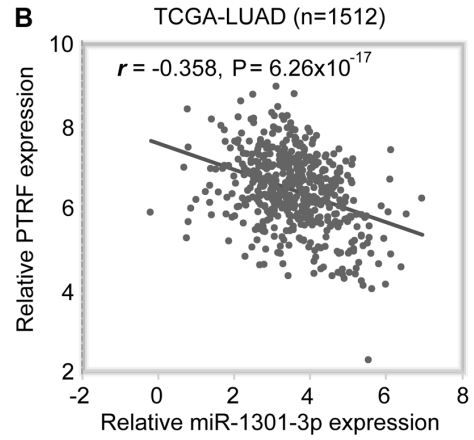

D

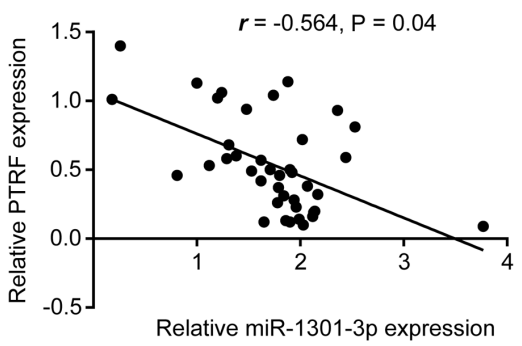

Figure 3. PTRF is a predicted target of miR-1301-3p. (A) The sequence alignment of PTRF 3'UTR and miR-1301-3p. (B) Bioinformatics analysis indicated that PTRF was inversely correlated with miR-1301-3p level in TCGA-LUAD dataset. (C) Bioinformatics analysis indicated that PTRF was inversely correlated with miR-1301-3p level in TCGA-LUSC dataset. (D) Pearson's correlation analysis was used to analyze the association between PTRF mRNA and miR-1301-3p expression in 40 lung tumors. LUSC, lung squamous cell carcinoma; LUAD, lung adenocarcinoma; PTRF, polymerase I and transcript release factor; miR, microRNA; TCGA, The Cancer Genome Atlas; 3'UTR, 3'untranslated region.

it was further demonstrated that the miR-1301-3p expression level was significantly negatively correlated with the PTRF mRNA expression level in 1,512 LUAD samples and 1,475 LUSC samples from TCGA-LUAD and TCGA-LUSC, respectively (Fig. 3B and C). Additionally, it was identified that there was a negative correlation between miR-1301-3p and PTFR mRNA level in the collected patient samples ( $n=40$; Fig. 3D).

Due to the higher expression of miR-1301-3p in A549 cells compared with that of H1299 cells, A549 were selected as a model to explore the molecular mechanism of miR-1301-3p in lung cancer. In A549 cells, miR-1301-3p inhibitor significantly increased PTRF mRNA expression level (Fig. 4A). Western blotting further demonstrated that miR-1301-3p inhibitor significantly increased PTRF protein level in A549 cells (Fig. 4B). Subsequently miR-1301-3p mimic was transfected into A549 cells to increase miR-1301-3p expression (Fig. 4C). Luciferase plasmids containing the 3'UTR of PTRF (PTRF 3'UTR-WT) or PTRF 3'UTR-Mut (with two-point mutations in the putative binding site) were constructed (Fig. 4D). Dual-luciferase reporter assay demonstrated that miR-1301-3p mimic significantly downregulated the luciferase activity of PTRF 3'UTR-WT, but not of PTRF 3'UTR-Mut in A549 cells (Fig. 4E).

miR-1301-3p regulates the expression of epithelial-mesenchymal transition (EMT) markers. PTRF is involved in the EMT process in lung cancer (20). In the present study, RT-qPCR demonstrated that miR-1301-3p inhibitor significantly increased E-cadherin and significantly decreased vimentin mRNA expression levels in A549 cells (Fig. 5A). Furthermore, western blotting revealed that miR-1301-3p inhibitor significantly increased E-cadherin and significantly decreased vimentin protein expression levels in A549 cells (Fig. 5B). These data indicated that miR-1301-3p regulates the EMT process in lung cancer.

Knockdown of PTRF attenuates the effect of miR-1301-3p downregulation in lung cancer. To evaluate the involvement of PTRF in miR-1301-3p-mediated cell proliferation and migration, PTRF siRNA was transfected into A549 cells. Western blotting confirmed that PTRF siRNA significantly decreased PTRF protein expression in A549 cells (Fig. 6A). By performing a CCK-8 assay, it was identified that PTRF-knockdown significantly reversed the effect of miR-1301-3p inhibitor on cell proliferation in A549 cells (Fig. 6B). Furthermore, in the migration assay, PTRF-knockdown significantly reversed the effect of miR-1301-3p on the migration of A549 cells (Fig. 6C). These data implied that miR-1301-3p facilitates cell proliferation and migration via targeting PTRF.

\section{Discussion}

A number of studies have suggested that miRNAs are involved in lung cancer progression $(4,5)$; however, the functions of numerous miRNAs have not yet been studied. The role of miR-1301-3p in human cancer is controversial. miR-1301-3p expression is lower in serum samples of patients with colorectal cancer compared with those from healthy volunteers (21). miR-1301-3p is downregulated in pancreatic adenocarcinoma (22). However, upregulation of miR-1301-3p has been observed in prostate cancer (23). The present study re-analyzed the previously published microarray data and discovered that miR-1301-3p is increased 

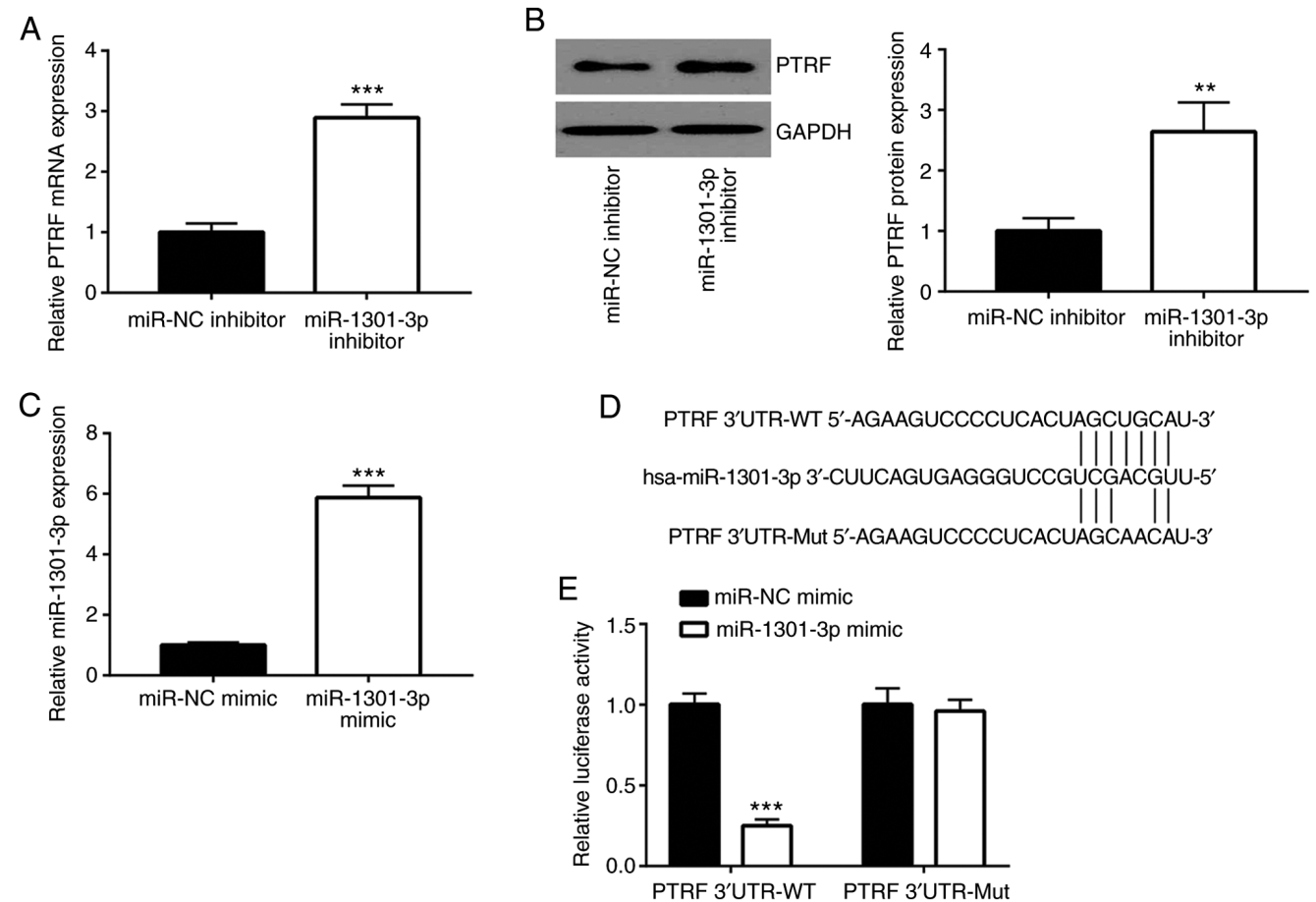

Figure 4. PTRF is directly targeted by miR-1301-3p in lung cancer cells. (A) RT-qPCR was used to measure PTRF mRNA levels in A549 cells after transfection of miR-1301-3p inhibitor or its NC. (B) Western blotting was performed to measure PTRF protein expression in A549 cells following transfection of miR-1301-3p inhibitor or its NC. (C) RT-qPCR was performed to detect miR-1301-3p expression in A549 cells transfected with miR-NC mimic or miR-1301-3p mimic. (D) Sequences of PTRF 3'UTR-WT, 3'UTR-Mut and miR-1301-3p. (E) Dual-luciferase reporter assay demonstrated that miR-1301-3p mimic repressed the relative luciferase activity of PTRF 3'UTR-WT in A549 cells. ${ }^{* *} \mathrm{P}<0.01,{ }^{* * * *} \mathrm{P}<0.001$ vs. miR-NC inhibitor. miR, microRNA; NC, negative control; PTRF, polymerase I and transcript release factor; RT-qPCR, reverse transcription-quantitative PCR; 3'UTR, 3'-untranslated region; WT, wild-type; mut, mutant.
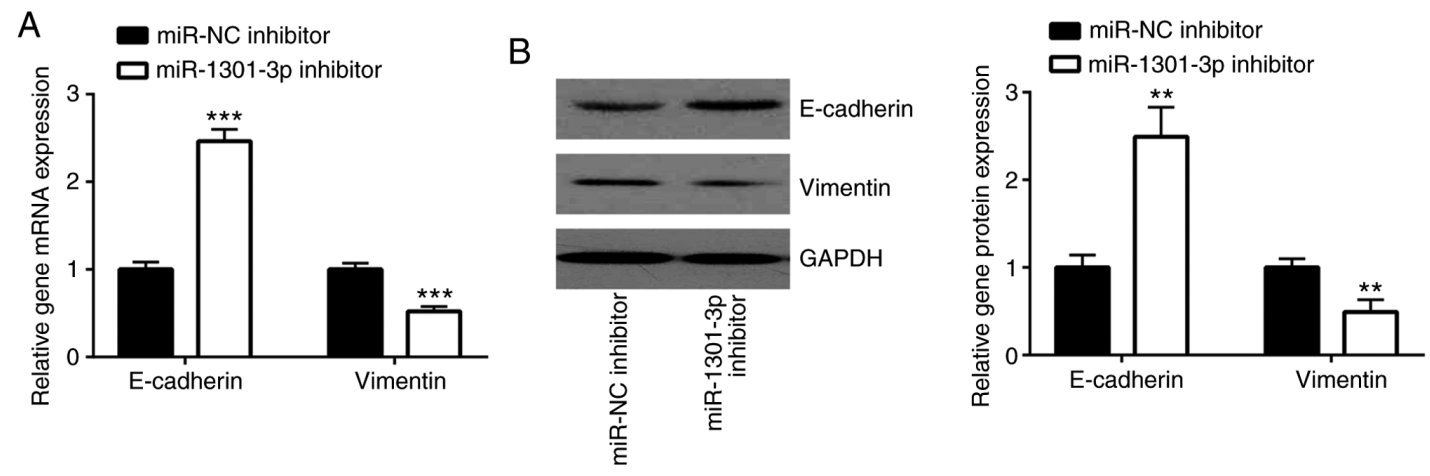

Figure 5. miR-1301-3p regulates the expression of key genes of the epithelial-mesenchymal transition. (A) Reverse transcription-quantitative PCR was performed to measure E-cadherin and Vimentin mRNA level in A549 cells following transfection with miR-1301-3p inhibitor or its NC. (B) Western blotting was performed to detect E-cadherin and Vimentin protein expression in A549 cells following transfection with miR-1301-3p inhibitor or its NC. ${ }^{* *} \mathrm{P}<0.01$, ${ }^{* * * *} \mathrm{P}<0.001$ vs. miR-NC inhibitor. miR, microRNA; NC, negative control.

in lung cancer with brain metastasis compared with those with non-brain metastasis. It was further confirmed that miR-1301-3p was increased in lung tumors compared with matched normal tissues. miR-1301-3p promotes or inhibits cell growth in different cell backgrounds. In prostate cancer, miR-1301-3p targets ubiquitination factor E4B, activates the p53 signaling pathway and suppresses cancer cell metastasis (24). It has also been reported that miR-1301-3p targets PPP2R2C and promotes anchorage-dependent and independent cell proliferation in prostate cancer cells (23). Using CCK- 8 and migration assays, the present study demonstrated that miR-1301-3p enhanced cell proliferation and migration of lung cancer. These data indicated that upregulation of miR-1301-3p may mediate lung cancer cell proliferation and migration. The current study evaluated the role of miR-1301-3p in lung cancer cells via in vitro assays. However, in vivo assays may provide several novel findings for investigating the function of miR-1301-3p in lung cancer. In the future, a mouse model will be used to study the involvement of miR-1301-3p in the initiation and development of lung cancer.

PTRF, also known as CAVIN1, is essential for the formation of caveolae (25). PTRF is a tumor suppressor in multiple cancer types (20,26-29). For example, PTRF overexpression restores caveolae formation, reduces matrix metalloproteinase-9 expression and inhibits the migration of 
A

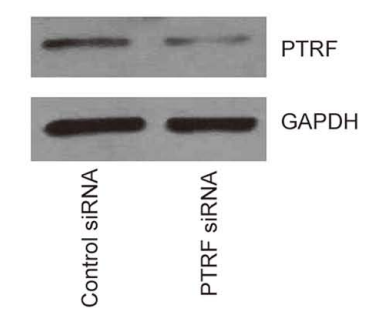

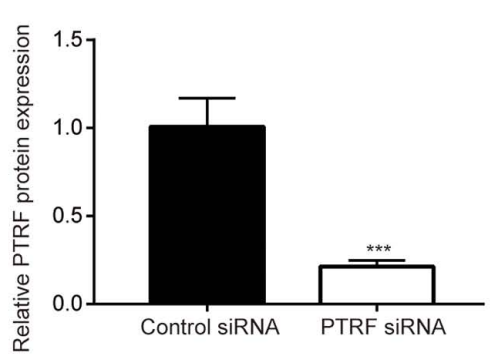

C

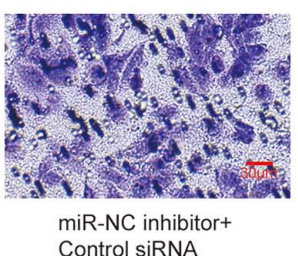

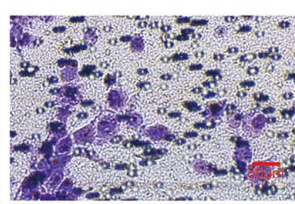

miR-1301-3p inhibitor+ Control siRNA

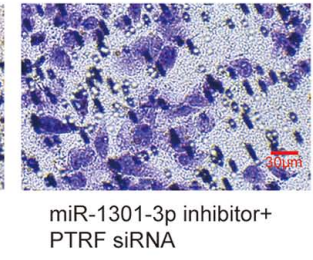

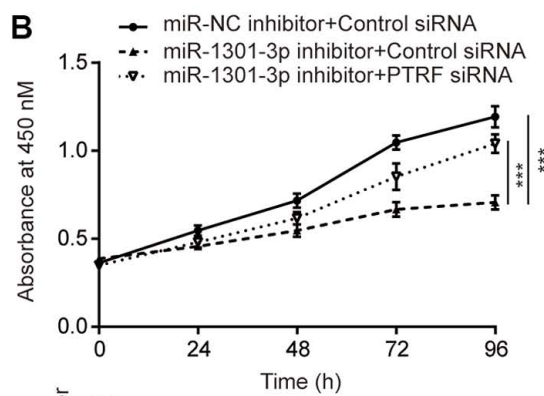

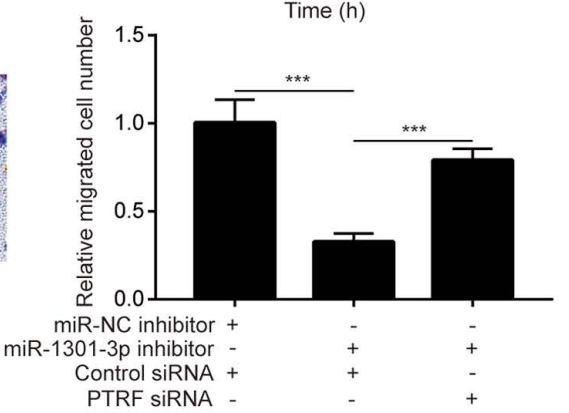

Figure 6. miR-1301-3p regulates lung cancer cell proliferation and migration via PTRF. (A) Western blotting was performed to measure PTRF protein level in A549 cells transfected with control siRNA or PTRF siRNA. ${ }^{* * * *} \mathrm{P}<0.001$ vs. control siRNA. (B) The Cell Counting Kit- 8 assay was performed to investigate proliferation of A549 cells after transfection with miR-1301-3p inhibitor or its NC in combination with control siRNA or PTRF siRNA. ${ }^{* * *} \mathrm{P}<0.001$. (C) The migration assay was performed to measure cell migration ability of A549 cells after transfection with miR-1301-3p inhibitor or its NC in combination with control siRNA or PTRF siRNA. ${ }^{* * *} \mathrm{P}<0.001$. miR, microRNA; NC, negative control; PTRF, polymerase I and transcript release factor; siRNA, small interfering RNA.

metastatic prostate cancer cells (29). In lung cancer, PTRF inhibits the EMT process and suppresses the cell proliferation and metastasis (20). The downregulation of PTRF is associated with dysregulation of miRNAs and DNA methylation in cancer cells $(20,30)$. Via bioinformatics analysis, the present study identified that PTRF is a potential target of miR-1301-3p. The expression of miR-1301-3p was shown to be negatively correlated with PTRF in TCGA-LUAD and TCGA-LUSC datasets. In addition, a dual-luciferase reporter assay confirmed that miR-1301-3p targeted PTRF in A549 cells, indicating miR-1301-3p is a novel regulator of PTRF. The EMT process is essential for the metastatic potential of lung cancer cells and is associated with dysregulation of miRNAs (31-33). PTRF inhibits the EMT process in cancer cells (20). Consistently, it was identified that miR-1301-3p regulated the expression of key genes involved in the EMT process. Furthermore, silencing of PTRF attenuated the effect of miR-1301-3p downregulation on A549 cells. The results indicated that the $\mathrm{miR}-1301-3 \mathrm{p} / \mathrm{PTRF}$ axis controls the EMT process and lung cancer cell metastasis. However, although the present study demonstrated a negative correlation between miR-1301-3p levels and PTRF mRNA levels, and it was experimentally revealed that miR-1301-3p targeted and repressed PTRF expression in lung cancer cells, several other mechanisms, such as post-translational modification may also regulate PTRF at the protein level. Future studies are needed to investigate the complex signaling network that regulates PTRF expression in lung cancer.

In conclusion, the current study revealed miR-1301-3p as a novel upregulated miRNA in lung cancer. miR-1301-3p was demonstrated to target PTRF and facilitate lung cancer cell proliferation and migration. Therefore, miR-1301-3p may be a promising biomarker for patients with lung cancer.

\section{Acknowledgements}

Not applicable.

\section{Funding}

The current study was funded by the National Youth Science Foundation (grant no. 81702252), the Healthy and Planning Commission Youth Program of Shanghai (grant no. 20164Y0160) and the Healthy and Planning Commission Program of Shanghai (grant no. 201840188).

\section{Availability of materials and data}

The datasets used and/or analyzed during the current study are available from the corresponding author on reasonable request. The results published here are in whole or part based upon data generated by the TCGA Research Network: https://www. cancer.gov/tcga.

\section{Authors' contributions}

YunW, QS, XC, YueW, YN and FL performed the experiments and analyzed the data. YunW and QS collected clinical samples. QS and FL were major contributors in conception, design and writing the manuscript. All authors read and approved the final manuscript.

\section{Ethics approval and consent to participate}

All participants provided written informed consent. The protocol of the experiments was approved by the Ethical Committee of Huadong Hospital Affiliated with Fudan University (Shanghai, China) (approval no. FDU201406-2). 


\section{Patient consent for participation}

Not applicable.

\section{Competing interests}

The authors declare that they have no competing interests.

\section{References}

1. Bray F, Ferlay J, Soerjomataram I, Siegel RL, Torre LA and Jemal A: Global cancer statistics 2018: GLOBOCAN estimates of incidence and mortality worldwide for 36 cancers in 185 countries. CA Cancer J Clin 68: 394-424, 2018.

2. Herbst RS, Morgensztern D and Boshoff C: The biology and management of non-small cell lung cancer. Nature 553: 446-454 2018.

3. Chen Z, Fillmore CM, Hammerman PS, Kim CF and Wong KK: Non-small-cell lung cancers: A heterogeneous set of diseases. Nat Rev Cancer 14: 535-546, 2014.

4. Hirono T, Jingushi K, Nagata T, Sato M, Minami K, Aoki M, Takeda AH, Umehara T, Egawa $\mathrm{H}$, Nakatsuji $\mathrm{Y}$, et al MicroRNA-130b functions as an oncomiRNA in non-small cel lung cancer by targeting tissue inhibitor of metalloproteinase-2. Sci Rep 9: 6956, 2019

5. Wang R, Chen XF and Shu YQ: Prediction of non-small cell lung cancer metastasis-associated microRNAs using bioinformatics. Am J Cancer Res 5: 32-51, 2014.

6. Yates LA, Norbury CJ and Gilbert RJ: The long and short of microRNA. Cell 153: 516-519, 2013.

7. Zhao Y and Srivastava D: A developmental view of microRNA function. Trends Biochem Sci 32: 189-197, 2007.

8. Bartel DP: MicroRNAs: Genomics, biogenesis, mechanism, and function. Cell 116: 281-297, 2004.

9. Wang Y, Liang Y and Lu Q: MicroRNA epigenetic alterations: Predicting biomarkers and therapeutic targets in human diseases. Clin Genet 74: 307-315, 2008.

10. Zhang B, Pan X, Cobb GP and Anderson TA: MicroRNAs as oncogenes and tumor suppressors. Dev Biol 302: 1-12, 2007.

11. Zhang Y, Sui J, Shen X, Li C, Yao W, Hong W, Peng H, Pu Y, Yin L and Liang G: Differential expression profiles of microRNAs as potential biomarkers for the early diagnosis of lung cancer. Oncol Rep 37: 3543-3553, 2017.

12. Tamiya H, Mitani A, Saito A, Ishimori T, Saito M, Isago H, Jo T, Yamauchi Y, Tanaka G and Nagase T: Exosomal microRNA expression profiling in patients with lung adenocarcinoma-associated malignant pleural effusion. Anticancer Res 38: 6707-6714, 2018.

13. Sun G, Ding X, Bi N, Wang Z, Wu L, Zhou W, Zhao Z, Wang J, Zhang W, Fan J, et al: Molecular predictors of brain metastasis-related microRNAs in lung adenocarcinoma. PLoS Genet 15: e1007888, 2019.

14. Mavridis K, Gueugnon F, Petit-Courty A, Courty Y, Barascu A, Guyetant S and Scorilas A: The oncomiR miR-197 is a novel prognostic indicator for non-small cell lung cancer patients. Br J Cancer 112: 1527-1535, 2015.

15. Zhu J, Wang S, Chen Y, Li X, Jiang Y, Yang X, Li Y, Wang X, Meng Y, Zhu M, et al: Mir-19 targeting of GSK3 $\beta$ mediates sulforaphane suppression of lung cancer stem cells. J Nutr Biochem 44: 80-91, 2017

16. Grosso S, Doyen J, Parks SK, Bertero T, Paye A, Cardinaud B, Gounon P, Lacas-Gervais S, Noël A, Pouysségur J, et al: MiR-210 promotes a hypoxic phenotype and increases radioresistance in human lung cancer cell lines. Cell Death Dis 4: e544, 2013.
17. Baumgartner U, Berger F, Hashemi Gheinani A, Burgener SS, Monastyrskaya K and Vassella E: miR-19b enhances proliferation and apoptosis resistance via the EGFR signaling pathway by targeting PP2A and BIM in non-small cell lung cancer. Mol Cancer 17: 44, 2018.

18. Livak KJ and Schmittgen TD: Analysis of relative gene expression data using real-time quantitative PCR and the 2(-Delta Delta C(T)) method. Methods 25: 402-408, 2001.

19. Zhou KR, Liu S, Cai L and Bin L: ENCORI: The encyclopedia of RNA interactomes.

20. Cai Y, Ruan J, Yao X, Zhao L and Wang B: MicroRNA-187 modulates epithelial-mesenchymal transition by targeting PTRF in non-small cell lung cancer. Oncol Rep 37: 2787-2794, 2017.

21. Wang L, Zhao Y, Xu M, Zhou F and Yan J: Serum miR-1301-3p, miR-335-5p, miR-28-5p, and their target B7-H3 may serve as novel biomarkers for colorectal cancer. J BUON 24: 1120-1127, 2019.

22. Dou D, Yang S, Lin Y and Zhang J: An eight-miRNA signature expression-based risk scoring system for prediction of survival in pancreatic adenocarcinoma. Cancer Biomark 23: 79-93, 2018.

23. Bi D, Ning H, Liu S, Que X and Ding K: miR-1301 promotes prostate cancer proliferation through directly targeting PPP2R2C. Biomed Pharmacother 81: 25-30, 2016.

24. Wang B, Wu H, Chai C, Lewis J, Pichiorri F, Eisenstat DD, Pomeroy SL and Leng RP: MicroRNA-1301 suppresses tumor cell migration and invasion by targeting the $\mathrm{p53} / \mathrm{UBE} 4 \mathrm{~B}$ pathway in multiple human cancer cells. Cancer Lett 401: 20-32, 2017.

25. Liu L and Pilch PF: A critical role of cavin (polymerase I and transcript release factor) in caveolae formation and organization. J Biol Chem 283: 4314-4322, 2008.

26. Gamez-Pozo A, Sanchez-Navarro I, Calvo E, Agulló-Ortuño MT, López-Vacas R, Díaz E, Camafeita E, Nistal M, Madero R, Espinosa E, et al: PTRF/cavin-1 and MIF proteins are identified as non-small cell lung cancer biomarkers by label-free proteomics. PLoS One 7: e33752, 2012.

27. Nassar ZD, Moon H, Duong T, Neo L, Hill MM, Francois M, Parton RG and Parat MO: PTRF/Cavin-1 decreases prostate cancer angiogenesis and lymphangiogenesis. Oncotarget 4: 1844-1855, 2013

28. Yi JS, Mun DG, Lee H, Park JS, Lee JW, Lee JS, Kim SJ, Cho BR, Lee SW and Ko YG: PTRF/cavin-1 is essential for multidrug resistance in cancer cells. J Proteome Res 12: 605-614, 2013.

29. Aung CS, Hill MM, Bastiani M, Parton RG and Parat MO: PTRF-cavin-1 expression decreases the migration of PC3 prostate cancer cells: Role of matrix metalloprotease 9. Eur J Cell Biol 90: 136-142, 2011.

30. Peng J, Liu HZ, Zhong J, Deng ZF, Tie CR, Rao Q, Xu W, You T, Li J, Cai CB, et al: MicroRNA187 is an independent prognostic factor in lung cancer and promotes lung cancer cell invasion via targeting of PTRF. Oncol Rep 36: 2609-2618, 2016.

31. Yochum ZA, Cades J, Wang H, Chatterjee S, Simons BW, O'Brien JP, Khetarpal SK, Lemtiri-Chlieh G, Myers KV, Huang EH, et al: Targeting the EMT transcription factor TWIST1 overcomes resistance to EGFR inhibitors in EGFR-mutant non-small-cell lung cancer. Oncogene 38: 656-670, 2019.

32. Dai L, Chen F, Zheng Y, Zhang D, Qian B, Ji H, Long F and Cretoiu D: miR-21 regulates growth and EMT in lung cancer cells via PTEN/Akt/GSK3 $\beta$ signaling. Front Biosci (Landmark Ed) 24: 1426-1439, 2019.

33. Amaar YG and Reeves ME: RASSF1C regulates miR-33a and EMT marker gene expression in lung cancer cells. Oncotarget 10: 123-132, 2019 .

(i) $\ominus$ This work is licensed under a Creative Commons Attribution-NonCommercial-NoDerivatives 4.0 International (CC BY-NC-ND 4.0) License. 